\title{
Synchronous B-Cell Acute Lymphoblastic Leukemia and Serous Ovarian Carcinoma: A Case Report
}

\author{
Michael Superdock ${ }^{a} \quad J_{\text {ustin Komisarof }}{ }^{a}$ Hani Katerji $^{b} \quad$ Eric Huselton ${ }^{a, c}$ \\ aDepartment of Medicine, University of Rochester Medical Center, Rochester, NY, USA; \\ ${ }^{b}$ Department of Pathology, University of Rochester Medical Center, Rochester, NY, USA; \\ 'Wilmot Cancer Institute, University of Rochester Medical Center, Rochester, NY, USA
}

\section{Keywords}

Leukemia · Ovarian carcinoma · Malignancy

\begin{abstract}
Adult patients with B-cell acute lymphoblastic leukemia (ALL) have higher rates of antecedent and subsequent malignancies. However, synchronous identification of ALL and ovarian cancer is exceedingly rare. We report the unique case of a 65 -year-old woman with synchronous B-cell ALL and low-grade serous ovarian carcinoma diagnosed after surgical intervention for a small bowel obstruction. Treatment with inotuzumab ozogamicin followed by adnexal mass resection and postoperative letrozole was successful in achieving complete remission for both her leukemia and ovarian cancer.
\end{abstract}

\section{Introduction}

Patients with B-cell ALL are at higher risk of developing a secondary primary malignancy $[1,2]$, and B-cell ALL occurs at higher rates in patients who have had an antecedent malignancy (am-ALL) $[3,4]$. In a patient with a new diagnosis of ALL, the simultaneous identification of a gynecological malignancy is particularly rare. Herein, we present the unique case of a 65-year-old female with no prior oncologic history who presented with B-cell ALL and serous ovarian carcinoma. We highlight the complicated hospital course that resulted in the 
Superdock et al.: Synchronous B-Cell Acute Lymphoblastic Leukemia and Serous
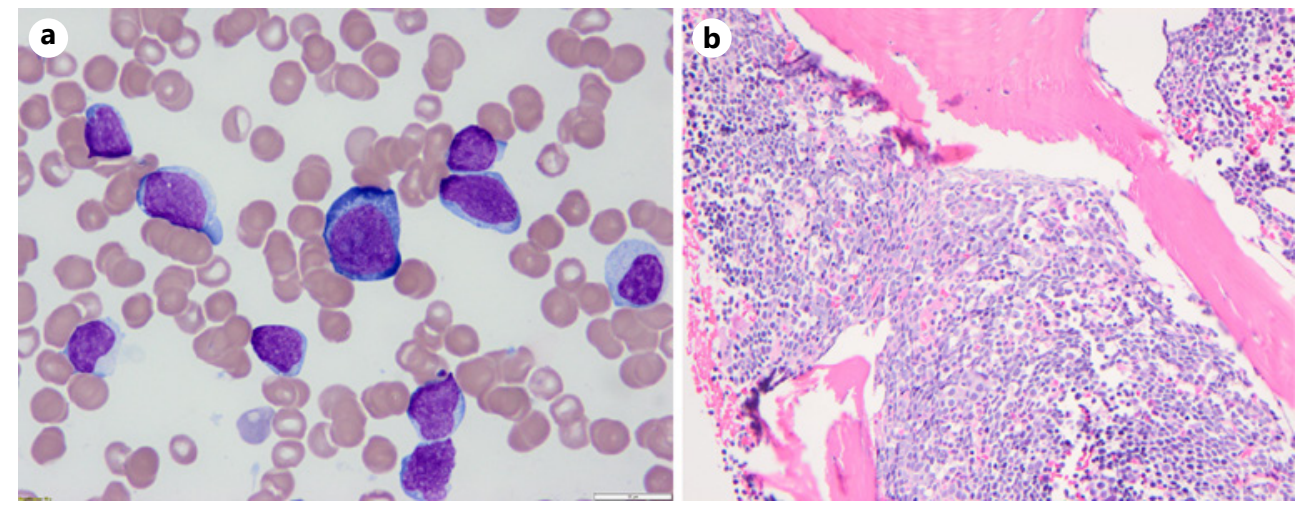

Fig. 1. a Wright and Giemsa-stained bone marrow aspirate showing numerous lymphoblasts with fine chromatin, prominent nucleoli, and scant to moderate cytoplasm. b Hematoxylin and eosin-stained bone marrow biopsy with near total replacement of the bone marrow space by lymphoblasts.

diagnosis of this pair of synchronous primary malignancies and discuss implications for prognosis and management.

\section{Case Report}

A 65-year-old female presented with two weeks of progressive dyspnea on exertion, associated with weakness, fatigue, and night sweats. She also reported several weeks of abdominal bloating, fullness, and early satiety. Her medical history was significant for atrial fibrillation previously treated with the maze procedure and mechanical mitral valve replacement on anticoagulation with warfarin. Prior surgeries included a hysterectomy and appendectomy. Family medical history was unknown as the patient was adopted.

Initial laboratory workup was significant for leukocytosis to $29.9 \mathrm{k} / \mu \mathrm{L}$ (normal range: $4.0-10.0 \mathrm{k} / \mu \mathrm{L}$ ) with $76 \%$ blasts as well as thrombocytopenia to $64 \mathrm{k} / \mu \mathrm{L}$ (normal range: $160-370$ $\mathrm{k} / \mu \mathrm{L}$ ). Peripheral blood smear (shown in Fig. 1) and flow cytometry sent on peripheral blood demonstrated B-cell ALL with the following immunophenotype: CD10 (bright), CD19, CD22 (dim), CD34, CD38, CD200, HLA-DR, cytoplasmic CD79a, and cytoplasmic TdT. Bone marrow biopsy confirmed the diagnosis with 83\% blasts. Karyotype was normal 46XX. Fluorescent in situ hybridization was positive for IgH rearrangement in $84.5 \%$ of cells and negative for BCR-ABL1, MLL, and ETV6/RUNX1 rearrangements. Lumbar puncture revealed no evidence of central nervous system disease.

CT scans obtained of the chest, abdomen, and pelvis identified bilateral adnexal complex cystic masses, with the largest mass on the left measuring $9.1 \times 5.5 \mathrm{~cm}$ (shown in Fig. 2). An adrenal nodule measuring $0.9 \times 1.1 \mathrm{~cm}$ was also noted, as well as splenomegaly measuring $16.0 \mathrm{~cm}$ in the craniocaudal direction. CA-125 level was markedly elevated at $475 \mathrm{U} / \mathrm{mL}$ (normal range: 0-34 U/mL). HE4, CA 19-9, and CEA levels were within normal limits. Given concern for a second malignancy, a core needle biopsy of the left ovarian mass was performed. Pathology of multiple soft tissue cores showed benign serous cystadenoma with no carcinoma or hematologic malignancy identified. Based on this initial workup and multidisciplinary consultation with hematology and obstetrics and gynecology, treatment of the B-cell ALL was prioritized.

The patient was enrolled in a clinical trial and treated with induction chemotherapy for B-cell ALL with the CD22 antibody-drug conjugate inotuzumab ozogamicin $0.8 \mathrm{mg} / \mathrm{m}^{2}$ on day 

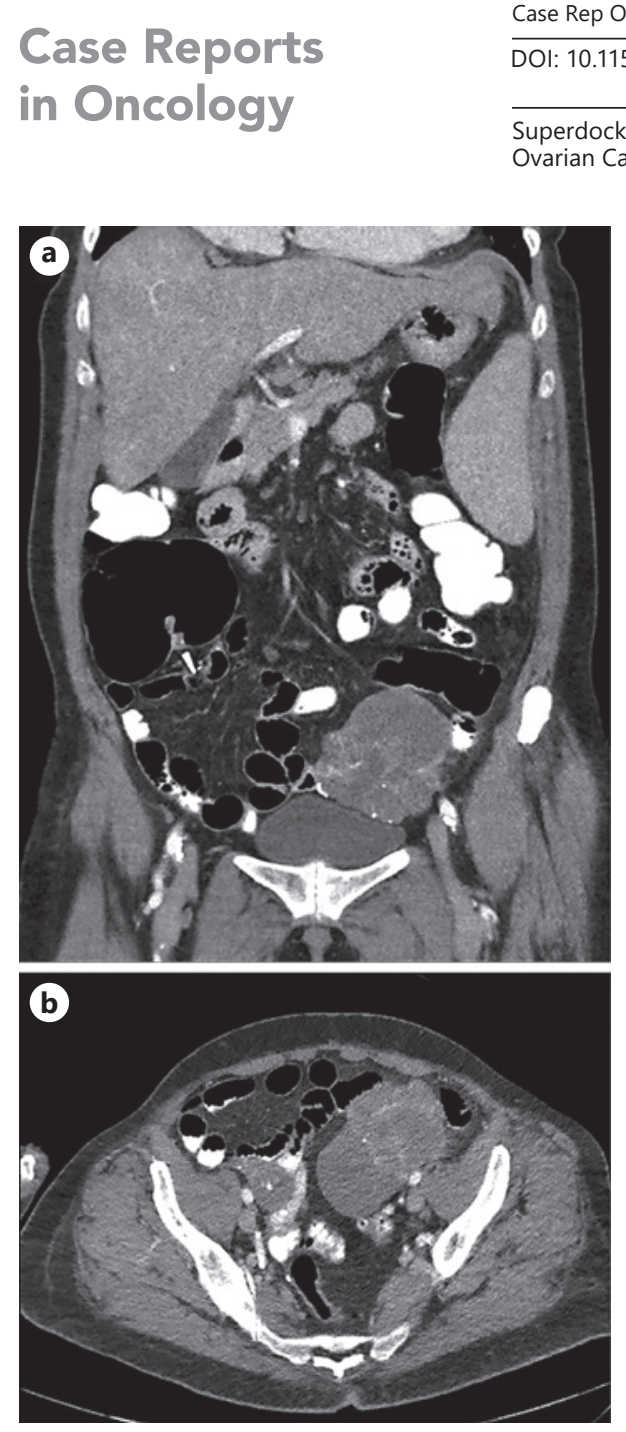

Fig. 2. a Coronal and $\mathbf{b}$ axial computed tomography images of the abdomen and pelvis visualized by soft tissue windows highlighting a complex cystic left adnexal mass measuring $9.1 \times 5.5 \mathrm{~cm}$.

1 followed by $0.5 \mathrm{mg} / \mathrm{m}^{2}$ on days 8 and 15 . She also received intrathecal methotrexate $(15 \mathrm{mg})$ for central nervous system prophylaxis. Bone marrow biopsy on day 18 revealed adequate cellularity and hematopoiesis with no evidence of leukemia. Minimal residual disease testing with flow cytometry revealed a small population of phenotypically abnormal B-cell precursors accounting for $0.021 \%$ of mononuclear cells with a similar immunophenotype.

During induction, the patient's course was complicated by progressive abdominal distension and discomfort. A second abdominal CT on day 19 revealed an incomplete small bowel obstruction. Despite bowel rest, the patient was unable to tolerate adequate oral intake. In consultation with gynecologic oncology and surgical oncology, further treatment for her ALL was delayed given her good response to inotuzumab ozogamicin in order to proceed with exploratory laparotomy. The patient subsequently underwent exploratory laparotomy on day 25 with lysis of adhesions, small bowel resection with primary anastomosis, pelvic lymphadenectomy, partial omentectomy, and bilateral salpingo-oophorectomy with adnexal mass resection. Pathology from bilateral adnexal masses, small bowel, and omentum was analyzed, with second opinions obtained from two distinct outside pathologists (shown in Fig. 3). The left ovarian tumor of size $15 \times 9 \mathrm{~cm}$ was classified as low-grade serous carcinoma in a background of serous borderline tumor/atypical proliferative serous tumor with extension into omentum by noninvasive desmoplastic implant and extension into small bowel serosa by noninvasive epithelial implant. The right ovarian tumor of size $4.5 \times 3.5 \mathrm{~cm}$ was classified as

\section{Karger'}




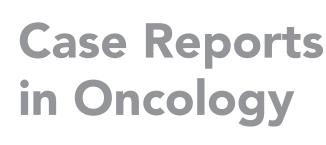

Case Rep Oncol 2021;14:1621-1626

\begin{tabular}{l|l}
\hline DOI: 10.1159/000519743 & ( 2021 The Author(s). Published by S. Karger AG, Basel
\end{tabular} www.karger.com/cro

Superdock et al.: Synchronous B-Cell Acute Lymphoblastic Leukemia and Serous Ovarian Carcinoma
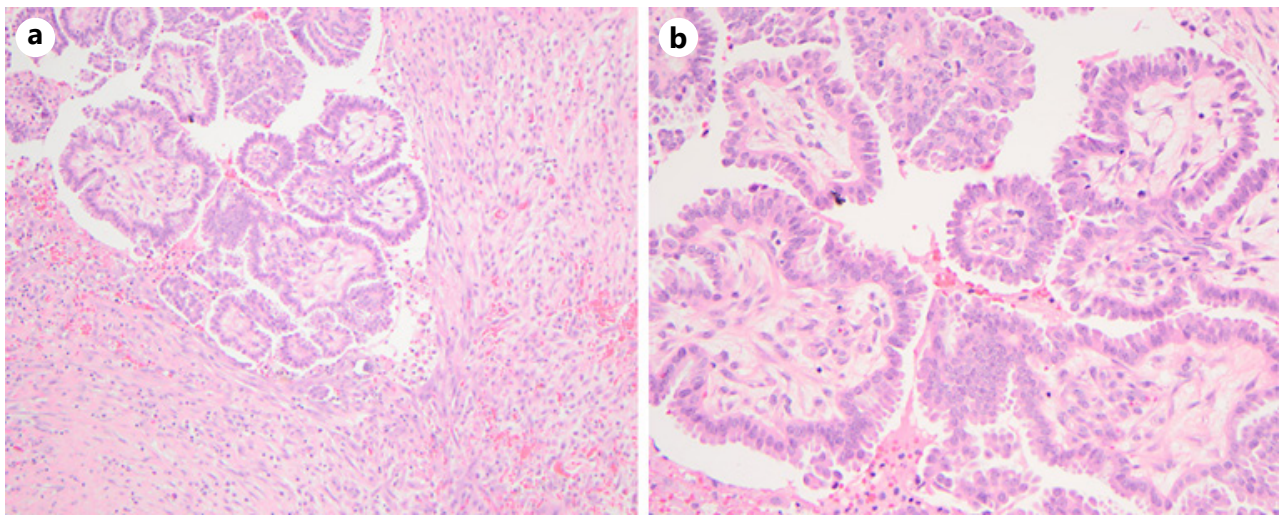

Fig. 3. a Hematoxylin and eosin-stained section of the left ovary showing low-grade serous carcinoma with invasive focus. b Higher magnification image of the left ovarian section highlighting the neoplastic papillary structures with small monotonous nuclei, low-grade atypia, and visible nucleoli.

serous borderline tumor/atypical proliferative serous tumor. Four pelvic lymph nodes were negative for malignancy. A final diagnosis of stage $2 \mathrm{~B}$ low-grade ovarian serous carcinoma of the L ovary was made.

Her postoperative course was complicated by ileus, deconditioning, and intra-abdominal fluid collections relieved by percutaneous drain placement. Given the delay in chemotherapy, a repeat bone marrow biopsy was performed 33 days after her last dose of inotuzumab ozogamicin. The biopsy revealed adequate cellularity and hematopoiesis with no evidence of leukemia. Minimal residual disease testing with flow cytometry was negative for residual B-cell ALL with a sensitivity of $1 / 10,000$ cells.

Following postoperative recovery, she was started on letrozole $2.5 \mathrm{mg}$ daily for her ovarian cancer and blinatumomab for consolidation of her B-cell ALL. Treatment with blinatumomab was complicated by grade 3 neurotoxicity which resolved with oral steroids. At 200 days after diagnosis, she remains in clinical remission from both her B-cell ALL and ovarian cancer, and her CA-125 has decreased to $31 \mathrm{U} / \mathrm{mL}$ (normal range: 0-34 U/mL).

\section{Discussion and Conclusion}

Synchronous presentation of acute lymphoblastic leukemia with any second primary malignancy is rare. To the best of our knowledge, this is the first case of synchronous adult ALL and ovarian cancer documented in the literature. Review of 9 registries of the Surveillance, Epidemiology, and End Results (SEER) database from 1975 to 2016 revealed no cases of ovarian cancer and ALL diagnosed in the same patient within a 1-year interval [5].

In our case, the diagnosis of B-cell ALL was made initially, and while there was suspicion for a secondary malignancy given the patient's bilateral adnexal masses and elevated CA-125, the diagnosis of serous ovarian carcinoma was confirmed only after exploratory laparotomy and adnexal mass resection for a small bowel obstruction. As a result, the malignancies were treated sequentially. Given that there is no consensus for optimal induction strategies for older adults with Philadelphia-chromosome negative B-cell ALL, the patient was enrolled in a clinical trial as recommended by NCCN guidelines. Induction with inotuzumab ozogamicin had the added benefit of sparing the patient from chemotherapy-induced thrombocytopenia, allowing her to continue therapeutic anticoagulation for her mechanical mitral valve. After the diagnosis of serous ovarian cancer was made, she underwent surgical resection and 
staging, followed by treatment with adjuvant letrozole. This sequence of treatments proved effective in achieving complete remission for her B-cell ALL and clinical remission for her serous ovarian carcinoma.

There is no known association between B-cell ALL and serous ovarian cancer. However, B-cell ALL in adults is known to occur at increased rates in individuals with an antecedent malignancy $[3,4]$ and is associated with greater risk of developing a second primary malignancy $[1,2]$. Although little is known about B-cell ALL that is synchronously diagnosed with a second primary malignancy, cases of adult ALL following an antecedent malignancy (am-ALL) have been well characterized.

Relative to de novo ALL, am-ALL has inferior overall survival $[3,4,6-8]$ and is associated with increased rates of high-risk cytogenic abnormalities including aberrations in the MLL gene, most commonly $\mathrm{t}(4 ; 11)$, as well as $\mathrm{t}(9 ; 22)$ and hypodiploidy $[3,9-13]$. These genetic abnormalities have been hypothesized to be associated with prior chemotherapy treatments [9-13]. However, when comparing patients with am-ALL who received prior treatment to those who have not, rates of overall survival and event-free survival are not significantly different $[10,14,15]$. These findings suggest that some cases of am-ALL may be due to an underlying predisposition to malignancy and possibly less so a consequence of prior antineoplastic therapy [15].

It is unclear whether adult ALL that is synchronously identified with a second malignancy, as in the case of our patient, is more akin to am-ALL or de novo ALL. This question is of particular relevance when considering prognosis, particularly in patients similar to ours, who otherwise lack the common high-risk cytogenic features commonly seen in am-ALL. Characterization of additional cases of ALL synchronously diagnosed with a second malignancy such as ovarian cancer could inform prognosis and approach to therapy in similar future cases.

\section{Statement of Ethics}

The Research Subjects Review Board of the University of Rochester reviewed this case report and concluded that the proposed activity is not research involving human subjects as defined by DHHS and FDA regulation. Approval was therefore not required. Written informed consent was obtained from the patient for publication of this case report and any accompanying images.

\section{Conflict of Interest Statement}

The authors report no conflicts of interest in this research.

\section{Funding Sources}

This project was supported by a Wilmot Cancer Research Fellowship grant.

\section{Author Contributions}

Michael Superdock, Justin Komisarof, and Eric Huselton reviewed the relevant scientific literature and wrote the original manuscript. Hani Katerji interpreted the pathology. All authors were involved in reviewing and editing the manuscript and figures. All authors approved the final manuscript.

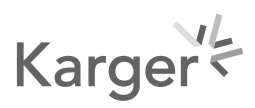




\section{Data Availability Statement}

The data that support the findings in this study are openly available in the Surveillance, Epidemiology, and End Results (SEER) database of the National Cancer Institute, which is made publicly available through www.seer.cancer.gov [5].

\section{References}

1 Shah BK, Ghimire KB. Second primary malignancies in adult acute lymphoblastic leukemia. Blood. 2013; 122(21):3871-1.

2 Tavernier E, Le QH, de Botton S, Dhédin N, Bulabois CE, Reman O, et al. Secondary or concomitant neoplasms among adults diagnosed with acute lymphoblastic leukemia and treated according to the LALA-87 and LALA-94 trials. Cancer. 2007 Dec;110(12):2747-55.

3 Rosenberg AS, Brunson A, Paulus JK, Tuscano J, Wun T, Keegan THM, et al. Secondary acute lymphoblastic leukemia is a distinct clinical entity with prognostic significance. Blood Cancer J. 2017 Sep;7(9):e605.

4 Swaika A, Frank RD, Yang D, Finn LE, Jiang L, Advani P, et al. Second primary acute lymphoblastic leukemia in adults: a SEER analysis of incidence and outcomes. Cancer Med. 2018 Feb;7(2):499-507.

5 National Cancer Institute. Surveillance, Epidemiology, and End results (SEER) program. Available from: www. seer.cancer.org. SEER*Stat Database: Incidence - SEER 9 Regs Research Data, Nov 2018 Sub (1975-2016) < Katrina/Rita Population Adjustment> - Linked to County Attributes - Total U.S., 1969-2017 Counties, National Cancer Institute, DCCPS, Surveillance Research Program, released April 2019, based on the November 2018 submission.

6 Ferraro F, Gao F, Stockerl-Goldstein K, Westervelt P, DiPersio JF, Ghobadi A. Secondary acute lymphoblastic leukemia, a retrospective analysis from Washington University and meta-analysis of published data. Leuk Res. 2018;72:86-91.

7 Giri S, Chi M, Johnson B, McCormick D, Jamy O, Bhatt VR, et al. Secondary acute lymphoblastic leukemia is an independent predictor of poor prognosis. Leuk Res. 2015 Dec;39(12):1342-6.

8 Shivakumar R, Tan W, Wilding GE, Wang ES, Wetzler M. Biologic features and treatment outcome of secondary acute lymphoblastic leukemia: a review of 101 cases. Ann Oncol. 2008 Sep;19(9):1634-8.

9 Ishizawa S, Slovak ML, Popplewell L, Bedell V, Wrede JE, Carter NH, et al. High frequency of pro-B acute lymphoblastic leukemia in adults with secondary leukemia with 11q23 abnormalities. Leukemia. 2003 Jun; 17(6):1091-5.

10 Tang G, Zuo Z, Thomas DA, Lin P, Liu D, Hu Y, et al. Precursor B-acute lymphoblastic leukemia occurring in patients with a history of prior malignancies: is it therapy-related? Haematologica. 2012 Jun;97(6):919-25.

11 Abdulwahab A, Sykes J, Kamel-Reid S, Chang H, Brandwein JM. Therapy-related acute lymphoblastic leukemia is more frequent than previously recognized and has a poor prognosis. Cancer. 2012 Aug;118(16):3962-7.

12 Aldoss I, Dagis A, Palmer J, Forman S, Pullarkat V. Therapy-related ALL: cytogenetic features and hematopoietic cell transplantation outcome. Bone Marrow Transplant. 2015 May;50(5):746-8.

13 Pagano L, Pulsoni A, Tosti ME, Annino L, Mele A, Camera A, et al. Acute lymphoblastic leukaemia occurring as second malignancy: report of the GIMEMA archive of adult acute leukaemia. Gruppo Italiano Malattie Ematologiche Maligne dell'Adulto. Br J Haematol. 1999 Sep;106(4):1037-40.

14 Pagano L, Pulsoni A, Tosti ME, Mele A, Mele L, Corvatta L, et al. Acute leukemia following a previous malignancy: do acute lymphoid leukemia and acute myeloid leukemia have common risk factors? Hematol J. 2000; 1(5):329-32.

15 Ganzel C, Devlin S, Douer D, Rowe JM, Stein EM, Tallman MS. Secondary acute lymphoblastic leukaemia is constitutional and probably not related to prior therapy. Br J Haematol. 2015 Jul;170(1):50-5. 